\title{
Heat risk exacerbation potential for neurology patients during the COVID-19 pandemic and related isolation
}

\author{
Alex Buoite Stella ${ }^{1}$ (I) $\cdot$ Davide Filingeri $^{2} \cdot$ Nicholas Ravanelli $^{3}$. Shawnda A. Morrison ${ }^{4} \cdot$ Miloš Ajčević $^{1,5}$. \\ Giovanni Furlanis ${ }^{1} \cdot$ Paolo Manganotti ${ }^{1}$
}

Received: 10 August 2020 / Revised: 9 October 2020 / Accepted: 29 October 2020 / Published online: 8 November 2020

(C) ISB 2020

\begin{abstract}
COVID-19 may increase the risk of heat-related symptoms during hot weather since vulnerable populations, including the elderly and those with neurological disabilities, must continue to self-isolate, often indoors. Within the chronic neurological patient population, indoor conditions in summer months present a hazard because of impaired and/or altered thermoregulation, including poor hydration status due to both autonomic and behavioral dysfunction(s). To address this increased risk, telemedicine protocols should include an assessment of the patient's environmental parameters, and when combined with physiological data from wearable devices, identify those with neurological diseases who are at higher risk of heat illness. Personalized medicine during times of self-isolation must be encouraged, and using smart technology in ambient assisted living solutions, including e-health to monitor physiological parameters are highly recommended, not only during extreme weather conditions but also during times of increased isolation and vulnerability.
\end{abstract}

Keywords COVID-19 $\cdot$ Neurological diseases $\cdot$ Physical distancing $\cdot$ Heat illness $\cdot$ Climate change $\cdot$ e-Health

Patients with neurological conditions are often at risk for suffering a variety of heat-related illnesses, especially when ambient temperatures are high. A recent World Meteorological Organization (WMO) and World Health Organization (WHO) promoted joint network (https://www.ghhin.org/ heat-and-covid-19) has determined that the indirect effect of extreme heat events will exacerbate patients' underlying conditions, especially considering the context of physical distancing and self-isolation measures imposed because of the ongoing COVID-19 pandemic. Indeed, isolation and

Alex Buoite Stella

abuoitestella@units.it

1 Clinical Unit of Neurology, Department of Medicine, Surgery and Health Sciences, Cattinara University Hospital ASUGI, University of Trieste, Strada di Fiume, 447, 34149 Trieste, Italy

2 THERMOSENSELAB, Environmental Ergonomics Research Centre, Loughborough University, Loughborough, UK

3 School of Kinesiology, Lakehead University, Thunder Bay, Canada

4 Faculty of Sport, University of Ljubljana, Ljubljana, Slovenia

5 Department of Engineering and Architecture, University of Trieste, Via Alfonso Valerio, 10, 34127 Trieste, Italy dependency are key factors associated with a higher risk of suffering heat-related illnesses during heatwaves (Belmin et al. 2007). Current directives to prevent the spread of COVID-19 may further restrict access to cooling spaces and/ or limit the use of air conditioning in public areas. A recent WHO technical report suggests that the most vulnerable populations include the elderly, those in self-isolation, and those with disabilities or pre-existing medical conditions such as cardiovascular, pulmonary, kidney, and metabolic diseases. Surprisingly, the report does not explicitly include those with neurological disorders in their list of pre-existing medical conditions. The COVID-19 global pandemic has already had a clear impact on the health of individuals diagnosed with neurological issues, requiring a reorganization of neurology units (Berardelli et al. 2020). This reorganization includes the use of digital technologies that allow for more remote clinical evaluations and follow-up for patients. However, there has not been enough emphasis placed on the unique, detrimental effects that additional heat, physical and social isolation, and a lack of access to quality medical care will have on those with chronic neurological conditions.

Amongst clinical populations, people with chronic neurological conditions are particularly vulnerable to hot weather because of well-documented autonomic and behavioral dysfunction(s), 


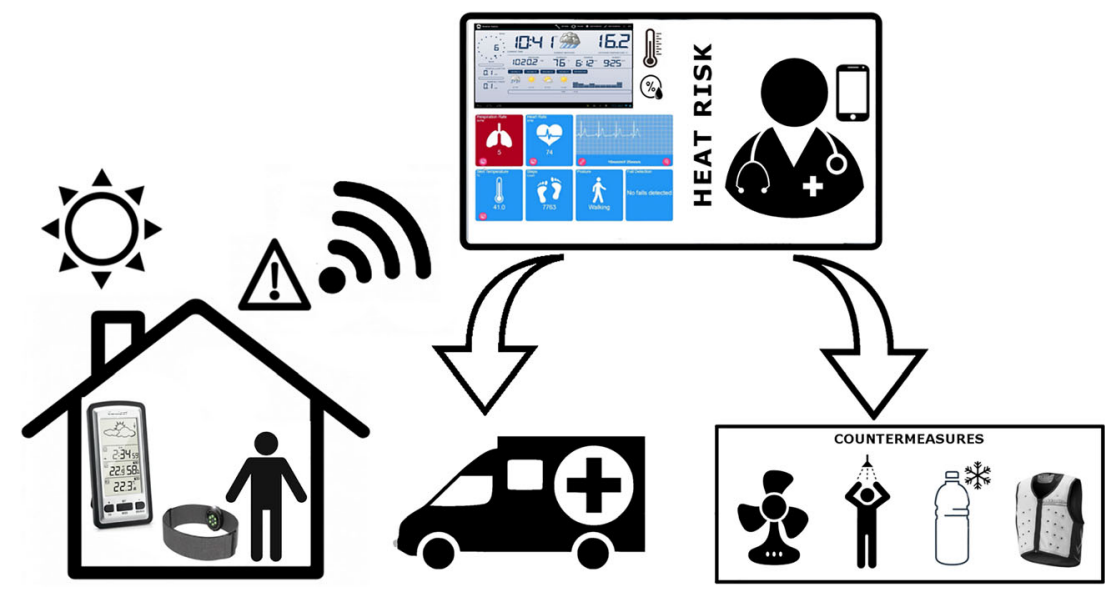

Fig. 1 Proposed protocol for heat symptoms prevention. Physiological (body temperature, heart rate, electrocardiography and respiratory rate, blood pressure, etc.) and environmental (temperature and humidity) data are collected and remotely accessible from the health service operators at the clinical center. If environmental conditions suggest a risk of heat symptoms, teleassistance protocol is activated and the operator or

impaired thermal sensation, cognitive/behavioral deficits, drugs consumption, or any combination thereof (Stöllberger et al. 2009). Considering this, each disease state is unique in the particular manifestation of their thermoregulatory deficiencies. For example, in Parkinson disease, altered central and peripheral thermoregulatory mechanisms, including deficits in sweating and vasomotor tone, and may contribute to the "parkinsonismhyperpyrexia syndrome" (Coon and Low 2018). Passive heat stress increases risk of orthostatic hypotension as tissues vasodilate, shunting blood from the core to periphery to cool the body (Lucas et al. 2012). Heat sensitivity is especially prevalent in those with multiple sclerosis, where 60 to $80 \%$ of people report worsening neurological symptoms as body temperature rises. Elevated body temperature typically occurs via a combination of warm environments (passive) and physical activity (active), which may predispose neurology patients to greater negative effects on overall health; excess heat also exacerbates fatigue, cognitive impairment, and balance loss (Christogianni et al. 2018). Motor neuron diseases, such as amyotrophic lateral sclerosis, are also at increased risk of heat-related illness due to a combination of altered thermoregulatory capacity in the severely disabled, and cognitive deficit (Dupuis et al. 2018). Stroke-induced deficits may predispose those to a higher risk of altered thermoregulation, especially in those with significant intraextracranial stenosis, and heat may influence cardio and cerebrovascular responses (Gostimirovic et al. 2020). The association of high ambient temperature and other neurological issues, like headache or seizures, should be further investigated since data on this topic are scarce. To compound the issue of overt heat illness, underhydration is a common feature in people with neurological conditions, both because of increased sweating rates during heat exposure and reduced fluid intake because of urinary incontinence or altered thirst sensation, further impairing patient physician can contact the patient, monitor physiological parameters, and according to heat risk prediction models, can activate the emergency system or suggest countermeasures to lower core temperature as (i) using a fan, (ii) wetting the skin, (iii) drinking cold water, and (iv) using cooling vests/garments

thermoregulation and increasing their heat-related symptoms. As such, close monitoring of fluid intake and hydration status should be encouraged in these special populations, especially during hot weather spells.

\section{Heat symptoms prevention protocol for neurologically impaired patients}

To reduce the risk of heat-related symptoms in patients with neurological disease, a novel protocol is proposed using teleassistance and telemonitoring programs with the support of wearable devices, as described in detail elsewhere (Furlanis et al. 2020). The protocol includes monitoring a trifecta of physiological parameters (heart rate, respiratory rate, and blood pressure) in addition to skin temperature, which have been more successful in determining whether people are suffering fever compared to models using only one physiological parameter (Sun et al. 2017; Jensen et al. 2019; Negishi et al. 2020; Buoite Stella et al. 2020a). Indeed, cardiorespiratory parameters have been used to develop prediction models of heat risk (Ravanelli et al. 2015; Tsuji et al. 2016; Laxminarayan et al. 2018) including for use in army personnel, which may yet be integrated to wearable technology (Buller et al. 2013; Chen et al. 2017). Home environmental parameters, such as temperature and humidity, would also be recorded and remotely monitored with a smart weather station. By setting alarms for environmental conditions based on known temperature thresholds (e.g., Pascal et al. 2013) and validated scales and models like the Universal Thermal Climate Index (UTCI) or the Heat Index (HI) (Katavoutas and Founda 2019), it would be possible to identify indoor environmental conditions which may predispose to a higher 
risk of heat stress. A further refinement would also include heat balance models, such as the Predictive Heat Strain (PHS; ISO 7933:2004) (Malchaire et al. 2001). These models are designed to assess hyperthermia risk in the general population, using input parameters like personal characteristics, physical activity, and environmental variables; the models can be applied (adapted, optimized) for neurological patients to prevent heat-related events from occurring in this population. Then, when a greater thermal challenge is identified by the net heat transfer capacity between human and environment, the patient would be contacted by their health service (Notley et al. 2018), and upon the discretion of the telemedicine physicians, countermeasures could be proposed to immediately lower body temperature. For example, in the absence of air conditioning, electric fan use under defined conditions and selfapplication of water to the skin surface have been shown to effectively lower cardiovascular strain, thermal discomfort, and sweating in healthy individuals exposed to extreme heat stress (Morris et al. 2019). Indeed, there are many viable cooling strategies available for individuals who present with altered thermoregulatory capacity. For example, in people with multiple sclerosis, drinking cold water (Chaseling et al. 2018) or wearing cooling garments (Buoite Stella et al. 2020b) does improve quality of life, exercise capacity, and reduces heat gain. The data collected from the project will serve as the foundation for at-home risk assessment for vulnerable populations and improve the delivery of telemedicine by obtaining a more complete physiological assessment (Fig. 1).

In summary, the COVID-19 pandemic has created a situation where there is an increased risk of heat illness, where patients with neurological conditions may be at greater risk. Since there is an urgent need to scale-up telemedicine practices and e-health solutions, specific protocols are needed to ensure all vulnerable populations remain at low risk for overt heat illness. In the absence of precise and validated noninvasive solutions to predict heat illness, the combination of physiological (cardiorespiratory, skin temperature) and environmental parameters may help to detect conditions predisposing to a heat risk, and promptly alert the telemedicine service. As such, "keeping a cool head and brain" during this pandemic, and in general during heatwaves, will be key to protecting people with neurological diseases from suffering further heat-related events, especially when this population has a demonstrated history of specific thermoregulatory needs compared to other high-risk health groups.

Acknowledgments The authors would like to thank Matteo di Franza for editorial and proofreading assistance. ABS received the unconditional support from the Merck Prize in Neurology. MA and GF received the unconditional support by Cloud Assisted for Health and Safety - CASSIA project (POR-FESR FVG 2014-2020).

\section{Compliance with ethical standards}

Conflict of interest The authors declare that they have no conflicts of interest.

Ethics and consents Not applied to this study.

\section{References}

Belmin J, Auffray J-C, Berbezier C, Boirin P, Mercier S, de Reviers B, Golmard JL (2007) Level of dependency: a simple marker associated with mortality during the 2003 heatwave among French dependent elderly people living in the community or in institutions. Age Ageing 36:298-303. https://doi.org/10.1093/ageing/afm026

Berardelli A, Silani V, Barone P, Calabresi P, Girlanda P, Lopiano L, Massacesi L, Monaco S, Onofrj M, Tassorelli C, Tedeschi G (2020) Neurology and the COVID-19 emergency. Neurol Sci 41:13431344. https://doi.org/10.1007/s10072-020-04465-8

Buller MJ, Tharion WJ, Cheuvront SN, Montain SJ, Kenefick RW, Castellani J, Latzka WA, Roberts WS, Richter M, Jenkins OC, Hoyt RW (2013) Estimation of human core temperature from sequential heart rate observations. Physiol Meas 34:781-798. https:// doi.org/10.1088/0967-3334/34/7/781

Buoite Stella A, Manganotti P, Furlanis G, et al (2020a) Return to school in the COVID-19 era: considerations for temperature measurement. J Med Eng Technol 1-4. https://doi.org/10.1080/03091902.2020. 1822941

Buoite Stella A, Pasquin F, Morrison SA, et al (2020b) Effects of a cooling vest with sham condition on walking capacity in heatsensitive people with multiple sclerosis. Eur J Appl Physiol 120: 2467-2476

Chaseling GK, Filingeri D, Barnett $M$ et al (2018) Cold water ingestion improves exercise tolerance of heat-sensitive people with MS. Med Sci Sports Exerc 50:643-648. https://doi.org/10.1249/MSS. 0000000000001496

Chen S-T, Lin S-S, Lan C-W, Hsu H-Y (2017) Design and development of a wearable device for heat stroke detection. Sensors (Basel) 18: 17. https://doi.org/10.3390/s18010017

Christogianni A, Bibb R, Davis SL et al (2018) Temperature sensitivity in multiple sclerosis: an overview of its impact on sensory and cognitive symptoms. Temp (Austin, Tex) 5:208-223. https://doi.org/10. 1080/23328940.2018.1475831

Coon EA, Low PA (2018) Thermoregulation in Parkinson disease. Handb Clin Neurol 157:715-725. https://doi.org/10.1016/B978-0444-64074-1.00043-4

Dupuis L, Petersen Å, Weydt P (2018) Thermoregulation in amyotrophic lateral sclerosis. Handb Clin Neurol 157:749-760. https://doi.org/ 10.1016/B978-0-444-64074-1.00046-X

Furlanis G, Ajčević M, Naccarato M et al (2020) e-Health vs COVID-19: home patient telemonitoring to maintain TIA continuum of care. Neurol Sci 41:2023-2024

Gostimirovic M, Novakovic R, Rajkovic J, Djokic V, Terzic D, Putnik S, Gojkovic-Bukarica L (2020) The influence of climate change on human cardiovascular function. Arch Environ Occup Health 75:19. https://doi.org/10.1080/19338244.2020.1742079

Jensen MM, Kellett JG, Hallas P, Brabrand M (2019) Fever increases heart rate and respiratory rate; a prospective observational study of acutely admitted medical patients. Acute Med 18:141-143

Katavoutas G, Founda D (2019) Intensification of thermal risk in Mediterranean climates: evidence from the comparison of rational and simple indices. Int J Biometeorol 63:1251-1264. https://doi.org/ 10.1007/s00484-019-01742-w

Laxminarayan S, Rakesh V, Oyama T, Kazman JB, Yanovich R, Ketko I, Epstein Y, Morrison S, Reifman J (2018) Individualized estimation of human core body temperature using noninvasive measurements. J 
Appl Physiol 124:1387-1402. https://doi.org/10.1152/japplphysiol. 00837.2017

Lucas RAI, Ainslie PN, Morrison SA, Cotter JD (2012) Compression leggings modestly affect cardiovascular but not cerebrovascular responses to heat and orthostatic stress in young and older adults. Age (Dordr) 34:439-449. https://doi.org/10.1007/s11357-011-9250-4

Malchaire J, Piette A, Kampmann B, Mehnert P, Gebhardt H, Havenith G, den Hartog E, Holmer I, Parsons K, Alfano G, Griefahn B (2001) Development and validation of the predicted heat strain model. Ann Occup Hyg 45:123-135

Morris NB, Gruss F, Lempert S, English T, Hospers L, Capon A, Jay O (2019) A preliminary study of the effect of dousing and foot immersion on cardiovascular and thermal responses to extreme heat. JAMA 322:1411-1413. https://doi.org/10.1001/jama.2019.13051

Negishi T, Abe S, Matsui T, Liu H, Kurosawa M, Kirimoto T, Sun G (2020) Contactless vital signs measurement system using RGBthermal image sensors and its clinical screening test on patients with seasonal influenza. Sensors (Basel) 20:2171. https://doi.org/10. $3390 / \mathrm{s} 20082171$

Notley SR, Flouris AD, Kenny GP (2018) On the use of wearable physiological monitors to assess heat strain during occupational heat stress. Appl Physiol Nutr Metab 43:869-881. https://doi.org/10. 1139/apnm-2018-0173

Pascal M, Wagner V, Le Tertre A et al (2013) Definition of temperature thresholds: the example of the French heat wave warning system. Int J Biometeorol 57:21-29. https://doi.org/10.1007/s00484-012-0530-1

Ravanelli NM, Hodder SG, Havenith G, Jay O (2015) Heart rate and body temperature responses to extreme heat and humidity with and without electric fans. JAMA 313:724-725. https://doi.org/10. 1001/jama.2015.153

Stöllberger C, Lutz W, Finsterer J (2009) Heat-related side-effects of neurological and non-neurological medication may increase heatwave fatalities. Eur J Neurol 16:879-882. https://doi.org/10. 1111/j.1468-1331.2009.02581.x

Sun G, Nakayama Y, Dagdanpurev S, Abe S, Nishimura H, Kirimoto T, Matsui T (2017) Remote sensing of multiple vital signs using a CMOS camera-equipped infrared thermography system and its clinical application in rapidly screening patients with suspected infectious diseases. Int J Infect Dis 55:113-117. https://doi.org/10.1016/j. ijid.2017.01.007

Tsuji B, Hayashi K, Kondo N, Nishiyasu T (2016) Characteristics of hyperthermia-induced hyperventilation in humans. Temp (Austin, Tex) 3:146-160. https://doi.org/10.1080/23328940.2016.1143760 